\title{
Usefulness of hexamethylenetetramine in combination with chemotherapy using free and pegylated liposomal doxorubicin in vivo, referring to the effect on quiescent cells
}

\author{
SHIN-ICHIRO MASUNAGA ${ }^{1}$, KENJI KONO $^{2}$, JUN NAKAMURA $^{3}$, \\ KEIZO TANO $^{4}$, HIROYUKI YOSHIDA ${ }^{2}$, MASAMI WATANABE ${ }^{4}$, GENRO KASHINO $^{1}$, \\ MINORU SUZUKI ${ }^{1}$, YUKO KINASHI ${ }^{5}$, YONG LIU ${ }^{1}$ and KOJI ONO ${ }^{1}$ \\ ${ }^{1}$ Particle Radiation Oncology Research Center, Research Reactor Institute, Kyoto University, Kumatori, Osaka 590-0494; \\ ${ }^{2}$ Department of Applied Chemistry, Graduate School of Engineering, Osaka Prefecture University, Naka-ku, Sakai, \\ Osaka 599-8531, Japan; ${ }^{3}$ Department of Environmental Sciences and Engineering, University of North Carolina \\ at Chapel Hill, Chapel Hill, NC 27599, USA; ${ }^{4}$ Particle Radiation Biology and ${ }^{5}$ Radiation Safety and Control, \\ Research Reactor Institute, Kyoto University, Kumatori, Osaka 590-0494, Japan
}

Received October 30, 2008; Accepted January 14, 2009

DOI: $10.3892 /$ or_00000355

\begin{abstract}
SCC VII tumor-bearing mice were continuously given 5-bromo-2'-deoxyuridine (BrdU) to label all intratumor proliferating $(\mathrm{P})$ cells. They received hexamethylenetetramine (HMTA) either once intraperitoneally or continuously subcutaneously together with chemotherapy using intraperitoneally administered free doxorubicin (DXR) or intravenously injected pegylated liposomal doxorubicin (PLD). One hour after the free DXR loading or $24 \mathrm{~h}$ after the PLD loading, the response of intratumor quiescent (Q) cells was assessed in terms of the micronucleus frequency using immunofluorescence staining for BrdU. The response of the total $(\mathrm{P}+\mathrm{Q})$ tumor cell population was determined from the tumors not treated with BrdU. Encapsulation of DXR into pegylated liposomes significantly enhanced cytotoxicity, especially in Q cells. HMTA, especially when administered continuously, efficiently increased the sensitivity to DXR, particularly in Q cells. The increase in sensitivity on the continuous rather than single administration of HMTA was a little clearer in the total cell population than in Q cells. DXR's encapsulation into pegylated liposomes and combination with HMTA, particularly when administered continuously, apparently reduced the difference in sensitivity to free DXR between the total and Q cell populations. In terms of the tumor cell-killing effect as a whole, including Q cells, the encap-
\end{abstract}

Correspondence to: Dr Shin-ichiro Masunaga, Particle Radiation Oncology Research Center, Research Reactor Institute, Kyoto University, 2-1010, Asashiro-nishi, Kumatori-cho, Sennan-gun, Osaka 590-0494, Japan

E-mail: smasuna@rri.kyoto-u.ac.jp

Key words: pegylated liposomal doxorubicin, hexamethylenetetramine, quiescent cell, hypoxia sulation of DXR into pegylated liposomes and combination with HMTA, particularly through continuous administration, are very promising, taking into account that HMTA has been used clinically.

\section{Introduction}

Pegylated liposomal doxorubicin (PLD) is a liposomal formulation of doxorubicin, reducing uptake by the reticuloendothelial system due to the attachment of polyethylene glycol polymers to a lipid anchor and stably retaining drugs as a result of liposomal entrapment via an ammonium sulfate chemical gradient (1). These features result in a pharmacokinetic profile characterized by an extended circulation time and a reduced volume of distribution, thereby promoting tumor uptake. Studies of tissue distribution indicated preferential accumulation into various implanted tumors and human tumor xenografts, with an enhancement of drug concentrations in the tumor when compared with conventional free doxorubicin (DXR) $(1,2)$. Clinical studies of PLD in humans have included patients with AIDS-related Kaposi's sarcoma and with a variety of solid tumors, including ovarian, breast and prostate carcinomas (2).

Formaldehyde preserves or fixes tissues or cells by irreversibly cross-linking primary amine groups in proteins with other nearby nitrogen atoms in protein or DNA through a -CH2- linkage (3). An acid-dependent formaldehyde donor, hexamethylenetetramine (HMTA) (Fig. 1), has been used as an antiseptic for urinary tract infections and characterized as a non-carcinogen in animals (3). Under hypoxic conditions in solid tumors, pyruvate generated by glycolysis creates a low $\mathrm{pH}$ environment that produces formaldehyde through dissociation of HMTA (4). Actually, based on the finding that HMTA released six molecules of formaldehyde in a $\mathrm{pH}$-dependent manner, DXR was shown to react with formaldehyde to yield an activated form of DXR that can further react with DNA to yield DXR-DNA adducts (4). 
Many cells in solid tumors are quiescent in situ but still clonogenic (5). Quiescent (Q) tumor cells are more resistant to radiation and conventional chemotherapy because of a larger hypoxic fraction and greater capacity to recover from potentially lethal damage than proliferating $(\mathrm{P})$ tumor cells. In this study, with our method for selectively detecting the response of Q cells within solid tumors (6), we investigated whether HMTA has selective killing effects on the intratumor hypoxia-rich $\mathrm{Q}$ cell population, compared with the total $(\mathrm{P}+\mathrm{Q})$ tumor cell population. In addition, the usefulness of continuous administration of HMTA was also evaluated (7). Actually, this study is one of the very few attempts to assess the effectiveness of HMTA as a systemic anti-tumor agent in vivo.

\section{Materials and methods}

Mice and tumors. SCC VII squamous cell carcinomas (Department of Radiotherapy, Kyoto University) derived from $\mathrm{C} 3 \mathrm{H} / \mathrm{He}$ mice were maintained in vitro in Eagle's minimum essential medium supplemented with $12.5 \%$ fetal bovine serum. The cells $\left(1.0 \times 10^{5}\right)$ were inoculated subcutaneously into both hind legs of 8- to 11-week-old syngeneic female C3H/He mice (Japan Animal Co., Ltd., Osaka, Japan). Fourteen days later, each tumor $\sim 1 \mathrm{~cm}$ in diameter was employed for experiments and the body weight of the tumorbearing mice was $22.1 \pm 2.3 \mathrm{~g}$ (mean $\pm \mathrm{SD}$ ). Mice were handled according to the Recommendations for Handling of Laboratory Animals for Biomedical Research, compiled by the Committee on Safety and Ethical Handling Regulations for Laboratory Animal Experiments, Kyoto University. Incidentally, the p53 of SCC VII tumor cells is the wild-type (8).

Labeling with 5-bromo-2'-deoxyuridine (BrdU). Nine days after the inoculation, mini-osmotic pumps (Durect Corporation, Cupertino, CA) containing BrdU dissolved in physiological saline $(250 \mathrm{mg} / \mathrm{ml})$ were implanted subcutaneously to label all $\mathrm{P}$ cells for 5 days. The labeling index after continuous labeling with BrdU was $55.3 \pm 4.5 \%$ and reached a plateau at this stage. Therefore, we regarded tumor cells not incorporating BrdU after continuous labeling as Q cells.

Preparation of pegylated liposomes encapsulating DXR (PLD). Liposome hydrogenated soy phosphatidylcholine (HSPC), cholesterol and distearoyl-phosphatidyl-ethanolamine conjugated at its amino position with a 2000 molecular weight segment of polyethylene glycol carbamate (PEG-2000-DSPE) $(56.7 / 38.2 / 5.1, \mathrm{~mol} \%)(145.8 \mathrm{mg})$ were dissolved in chloroform and the solvent was removed by evaporation. The obtained thin lipid/copolymer-mixed membrane was further dried under vacuum for $3 \mathrm{~h}$ and was dispersed in an aqueous $\left(\mathrm{NH}_{4}\right)_{2} \mathrm{SO}_{4}$ solution (300 mM, $9 \mathrm{ml}$, pH 5.3). The liposome suspension was extruded through a polycarbonate membrane with a pore size of $100 \mathrm{~nm}$ and was applied to a Sepharose 4B column equilibrated with $20 \mathrm{mM}$ Hepes and $150 \mathrm{mM} \mathrm{NaCl}$ (pH 7.4). Then, an aqueous DXR solution $(10 \mathrm{mg} / \mathrm{ml}, 390 \mathrm{ml})$ was added to the recovered liposome suspension $(13.9 \mathrm{ml}, 1.7 \mathrm{mM}$ of HSPC) and the mixed suspension was incubated for $1 \mathrm{~h}$ at $60^{\circ} \mathrm{C}$. The DXR-loaded liposomes were again purified using a Sepharose 4B column with a mixture of $20 \mathrm{mM}$ Hepes and

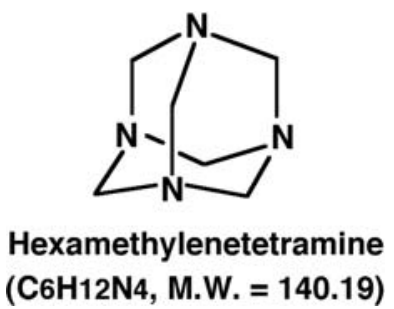

Figure 1. Chemical structure of hexamethylenetetramine (HMTA).

$150 \mathrm{mM} \mathrm{NaCl}$ (pH 7.4) (9). Mean particle size was $138 \mathrm{~nm}$. DXR was obtained from Kyowa Hakko Kogyo Co., Ltd, Japan.

Treatment. After the labeling with BrdU, HMTA dissolved in physiological saline was administered at a dose of 28.5 millimole $/ \mathrm{kg}$, singly by intraperitoneal injection or continuously for $24 \mathrm{~h}$ by subcutaneously implanting miniosmotic pumps. The dose employed, yielding a surviving fraction of $\sim 0.5$ on the single intraperitoneal administration of HMTA only, was based on our previous study (10) and the toxicity data on the Material Safety Data Sheet for HMTA (intravenous, mouse, $\mathrm{LD}_{50}=9200 \mathrm{mg} / \mathrm{kg}$ ). One hour after the intraperitoneal injection or after the 24-h continuous subcutaneous infusion of HMTA, free DXR and PLD were administered at a dose of 2.0 through $8.0 \mathrm{mg} / \mathrm{kg}$ (as a concentration of DXR) intraperitoneally and intravenously through the tail vein, respectively. The maximum tolerated dose of free DXR is $8 \mathrm{mg} / \mathrm{kg}$. One hour after the intraperitoneal administration of free DXR or $24 \mathrm{~h}$ after the intravenous administration of PLD, implanted tumors were excised. All above-mentioned sequences and the timing of each treatment were appropriate enough to function completely $(1,2)$.

Immunofluorescence staining of BrdU-labeled cells and micronucleus (MN) assay. Tumors excised from the mice given BrdU were minced and trypsinized $[0.05 \%$ trypsin and $0.02 \%$ ethylenediamine-tetraacetic acid (EDTA) in phosphatebuffered saline (PBS), $37^{\circ} \mathrm{C}, 15 \mathrm{~min}$ ]. Tumor cell suspensions were incubated for $72 \mathrm{~h}$ in tissue culture dishes containing complete medium and $1.0 \mu \mathrm{g} / \mathrm{ml}$ of cytochalasin-B to inhibit cytokinesis while allowing nuclear division and the cultures were then trypsinized and cell suspensions were fixed. After the centrifugation of fixed cell suspensions, the cell pellet was resuspended with cold Carnoy's fixative (ethanol:acetic acid $=3: 1$ in volume). The suspension was then placed on a glass microscope slide and the sample was dried at room temperature. The slides were treated with $2 \mathrm{M}$ hydrochloric acid for $60 \mathrm{~min}$ at room temperature to dissociate the histones and partially denature the DNA. The slides were then immersed in borax-borate buffer $(\mathrm{pH} 8.5)$ to neutralize the acid. BrdU-labeled tumor cells were detected by indirect immunofluorescence staining using monoclonal anti-BrdU antibody (Becton-Dickinson, San Jose, CA) and fluorescein isothiocyanate (FITC)-conjugated anti-mouse IgG antibody (Sigma, St. Louis, MO). To observe the double staining of tumor cells with green-emitting FITC and red-emitting propidium iodide (PI), cells on the slides were treated with PI $(2 \mu \mathrm{g} / \mathrm{ml}$ in PBS $)$ and monitored under a fluorescence microscope. 
Table I. Plating efficiency and micronucleus frequency without doxorubicin administration.

Total tumor cells Quiescent cells

\begin{tabular}{lcc}
\hline Plating efficiency & & \\
Without HMTA & $52.0 \pm 4.5^{\mathrm{a}}$ & - \\
With HMTA i.p. & $25.4 \pm 2.7$ & - \\
With HMTA cont. & $18.2 \pm 1.9$ & - \\
Micronucleus frequency & & \\
Without HMTA & $0.056 \pm 0.005$ & $0.081 \pm 0.011$ \\
With HMTA i.p. & $0.193 \pm 0.014$ & $0.261 \pm 0.025$ \\
With HMTA cont. & $0.287 \pm 0.029$ & $0.383 \pm 0.034$ \\
\hline
\end{tabular}

HMTA, hexamethylenetetramine; i.p., single intraperitoneal administration; cont., continuous subcutaneous administration and ${ }^{a}$ mean \pm standard deviation.

The MN frequency in cells not labeled with BrdU could be examined by counting the micronuclei in the binuclear cells that showed only red fluorescence. The MN frequency was defined as the ratio of the number of micronuclei in the binuclear cells to the total number of binuclear cells observed (6). The MN frequency was also shown to be a tool for detecting sensitivity to chemotherapeutic agents (6).

The ratios obtained in tumors not pretreated with BrdU indicated the MN frequency at all phases in the total tumor cell population. More than 400 binuclear cells were counted to determine the MN frequency.

Clonogenic cell survival assay. The clonogenic cell survival assay was also performed in the mice given no BrdU using an in vivo-in vitro assay method. Tumors were disaggregated by stirring for $20 \mathrm{~min}$ at $37^{\circ} \mathrm{C}$ in PBS containing $0.05 \%$ trypsin and $0.02 \%$ EDTA. The cell yield was $(4.5 \pm 1.1) \times 10^{7} / \mathrm{g}$ tumor weight.

More than 3 tumor-bearing mice with a tumor at each hind leg were used to assess each set of conditions and each experiment was repeated twice. Namely, $>6$ mice $(=12$ tumors) were used for each set of conditions. To examine the differences between pairs of values, Student's t-test was used when variances of the two groups could be assumed to be equal; otherwise the Welch t-test was used. P-values are from two-sided tests.

\section{Results}

The plating efficiency and MN frequency when no dose of DXR was administered are shown in Table I. Plating efficiency in the total cell population and $\mathrm{MN}$ frequency in both the total and Q cell populations decreased and increased significantly $(\mathrm{P}<0.05)$, respectively, in the following order; without HMTA, with HMTA injected once intraperitoneally and with HMTA infused continuously subcutaneously. The values of MN frequency were significantly larger in the $\mathrm{Q}$ cell population than in the total cell population under all conditions $(\mathrm{P}<0.05)$.

The clonogenic cell survival curves for total tumor cell populations after free DXR (left panel) or PLD (right panel)

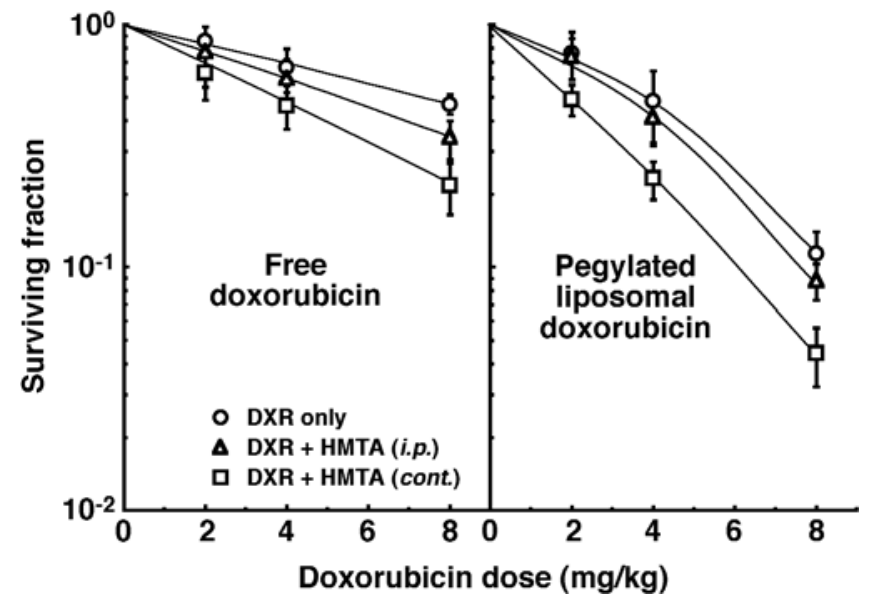

Figure 2. The clonogenic cell survival curves for the total tumor cell population after free doxorubicin (DXR) (left panel) or pegylated liposomal doxorubicin (PLD) (right panel) treatment with or without pre-treatment hexamethylenetetramine (HMTA) administration. HMTA was administered once intraperitoneally (i.p.) or continuously (cont.) subcutaneously for $24 \mathrm{~h}$. Bars represent standard deviations.

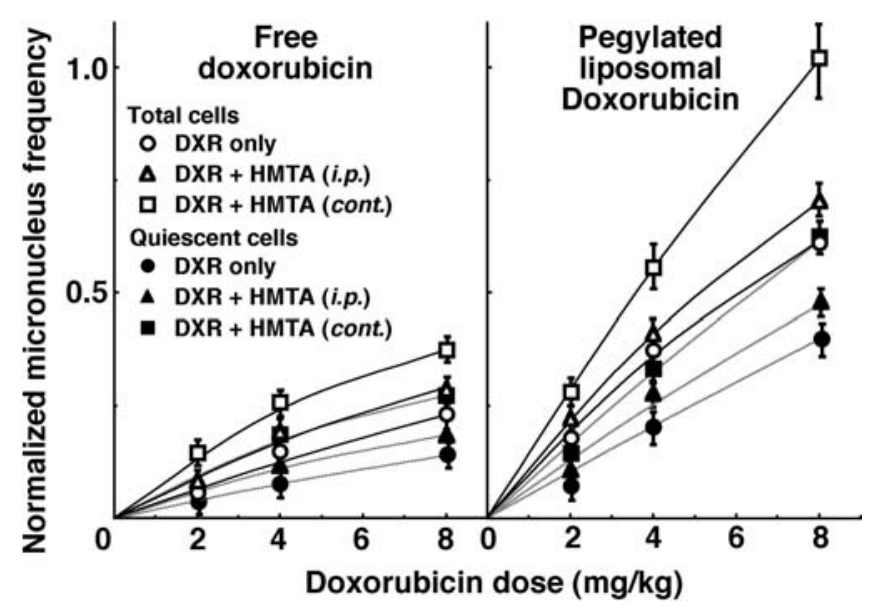

Figure 3. The normalized micronucleus frequencies of total and quiescent cell populations after free doxorubicin (DXR) (left panel) or pegylated liposomal doxorubicin (PLD) (right panel) treatment with or without pretreatment hexamethylenetetramine (HMTA) administration. HMTA was administered once intraperitoneally (i.p.) or continuously (cont.) subcutaneously for $24 \mathrm{~h}$. Bars represent standard deviations.

treatment with or without pre-treatment HMTA administration are shown in Fig. 2. Overall, the sensitivity to DXR was significantly increased with PLD rather than free DXR for all conditions $(\mathrm{P}<0.05)$. Whether free DXR or PLD was employed, the sensitivity to DXR was elevated in the following order; without HMTA < with HMTA injected once intraperitoneally $<$ with HMTA infused continuously subcutaneously.

For baseline correction, we used the normalized MN frequency to exclude the MN frequency in tumors not treated with DXR. The normalized MN frequency was the MN frequency in the tumors treated with free DXR or PLD minus that in the tumors not treated with DXR. The normalized MN frequencies of total and Q cell populations after free DXR (left panel) or PLD (right panel) treatment with or without pre-treatment HMTA administration are shown in Fig. 3. On the whole, in both populations, the sensitivity to DXR was 
Table II. Enhancement due to encapsulation into pegylated liposomes. ${ }^{a}$
DXR only
DXR+HMTA i.p.
DXR+HMTA cont.

Surviving fraction $=0.5$
Total cells

$1.9(1.8-2.0)$

$1.8(1.7-1.9)$

Normalized micronucleus frequency $=0.1$

Total cells

Quiescent cells

$3.0(2.9-3.1)$

$2.4(2.3-2.5)$

$2.2(2.1-2.3)$

DXR, doxorubicin; HMTA, hexamethylenetetramine; i.p., single intraperitoneal administration; cont., continuous subcutaneous administration.

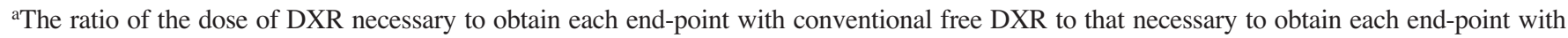
DXR encapsulated into pegylated liposomes. ${ }^{b}$ Values in parentheses are $95 \%$ confidence limits, determined using standard errors. When the ranges of $95 \%$ confidence limits do not overlap between any two values, the difference between the values is considered significant $(\mathrm{P}<0.05)$.

Table III. Enhancement due to combination with hexamethylenetetramine. ${ }^{\mathrm{a}}$

\begin{tabular}{lcccc}
\hline & \multicolumn{2}{c}{ Conventional free DXR } & & \multicolumn{2}{c}{ Pegylated liposomal DXR } \\
\cline { 2 - 3 } & + HMTA i.p. & + HMTA cont. & + HMTA i.p. & + HMTA cont. \\
\hline $\begin{array}{l}\text { Surviving fraction }=0.5 \\
\text { Total cells }\end{array}$ & $1.4(1.3-1.5)^{\mathrm{b}}$ & $2.0(1.9-2.1)$ & $1.15(1.1-1.2)$ & $1.65(1.55-1.75)$ \\
Normalized micronucleus frequency $=0.1$ & & & & \\
$\quad \begin{array}{l}\text { Total cells } \\
\text { Quiescent cells }\end{array}$ & $1.35(1.25-1.45)$ & $2.0(1.9-2.1)$ & $1.15(1.1-1.2)$ & $1.55(1.45-1.65)$ \\
\end{tabular}

DXR, doxorubicin; HMTA, hexamethylenetetramine; i.p., single intraperitoneal administration; cont., continuous subcutaneous administration.

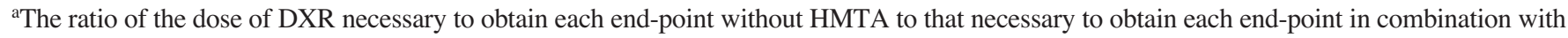
HMTA. ${ }^{b}$ Values in parentheses are $95 \%$ confidence limits, determined using standard errors. When the ranges of $95 \%$ confidence limits do not overlap between any two values, the difference between the values is considered significant $(\mathrm{P}<0.05)$.

significantly increased by PLD more than free DXR under all conditions $(\mathrm{P}<0.05)$. Whether free DXR or PLD was employed, in total and Q cell populations, the sensitivity increased in the following order; without HMTA < with HMTA injected once intraperitoneally $<$ with HMTA infused continuously subcutaneously.

To analyze the effect due to encapsulation into pegylated liposomes, DXR dose-modifying factors for the administration of PLD compared with free DXR were calculated using the data shown in Figs. 2 and 3 (Table II). In both total and Q cell populations, the sensitivity to DXR was significantly enhanced $>2$-fold by the encapsulation $(\mathrm{P}<0.05)$, with or without HMTA. However, the enhancement ratios were decreased, more dramatically in Q cells, in the following order; without HMTA $>$ with HMTA injected once intraperitoneally > with HMTA infused continuously subcutaneously.

To investigate the effect of a single intraperitoneal injection or the continuous subcutaneous infusion of HMTA, dose-modifying factors with versus without HMTA were calculated using the data shown in Figs. 2 and 3 (Table III). Whether free DXR or PLD was employed, in both total and Q cell populations, sensitivity to DXR was significantly enhanced $(\mathrm{P}<0.05)$ in the following order; without HMTA < with HMTA injected once intraperitoneal < with HMTA infused continuously subcutaneously. The enhancement was more marked in the Q cell population and with the use of free DXR than in the total cell population and with the use of PLD, respectively. The enhancement due to continuous administration compared with a single administration was more prominent in the total cell population and with the use of free DXR than in the Q cell population and with the use of PLD, respectively.

To examine the difference in sensitivity between the total and Q cell populations, dose-modifying factors, which compare the doses of DXR necessary to obtain a normalized $\mathrm{MN}$ frequency of 0.1 in $\mathrm{Q}$ cells with those in the total cell population, were calculated using the data in Figs. 2 and 3 (Table IV). Overall, the difference in sensitivity was suppressed on the use of PDL compared with free DXR. Whether free DXR or PLD was employed, the difference in sensitivity was reduced in the following order; without HMTA > with HMTA injected once intraperitoneally $>$ with HMTA infused continuously subcutaneously.

\section{Discussion}

Formaldehyde is a naturally occurring biological compound present in all tissues, cells and bodily fluids (3). It functions 
Table IV. Dose-modifying factors for quiescent cells relative to the total tumor cell population. ${ }^{\mathrm{a}}$

DXR only DXR+HMTA i.p. DXR+HMTA cont.

Normalized micronucleus frequency $=0.1$

\begin{tabular}{lccr} 
Conventinal free DXR & $1.9(1.75-2.05)^{\mathrm{b}}$ & $1.7(1.6-1.8)$ & $1.6(1.5-1.6)$ \\
Pegylated liposomal DXR & $1.75(1.65-1.85)$ & $1.6(1.5-1.7)$ & $1.5(1.4-1.6)$ \\
\hline
\end{tabular}

DXR, doxorubicin; HMTA, hexamethylenetetramine; i.p., single intraperitoneal administration; cont., continuous subcutaneous administration. ${ }^{a}$ The ratio of the dose of DXR necessary to obtain each normalized micronucleus frequency in quiescent cells to that necessary to obtain each

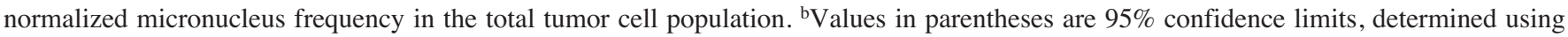
standard errors. When the ranges of $95 \%$ confidence limits do not overlap between any two values, the difference between the values is considered significant $(\mathrm{P}<0.05)$

as a key intermediate in the 'one-carbon pool' used for the biosynthesis of purines, thymidine and some amino acids. It is usually rapidly metabolized by reduction, oxidation and reduced glutathione-dependent pathways (3). However, saturation in formaldehyde metabolism may lead to DNA damage. It has been shown that cells exposed to formaldehyde exhibit, as a major form of DNA damage, DNA-protein crosslinks (11). In a limited oxygen environment, pyruvate generated by glycolysis in the cell cytoplasm is preferentially converted into lactic acid by lactate dehydrogenase, which creates a low $\mathrm{pH}$ environment $(\mathrm{pH}$ 6.4-6.8) (12). Further, through the Warburg effect, many cancer cells vigorously consume glucose and preferentially produce lactic acid even in the presence of adequate oxygen (12). Thus, due to the extracellular acidic conditions adjacent to solid tumors, an acid-dependent formaldehyde donor, HMTA, should dissociate to release formaldehyde into nearby tissues. Moreover, HMTA has been examined as an antiseptic for the treatment of urinary tract infections, as well as being studied in patients with maxillofacial phelegmons and as a prophylactic agent against recurrent acute cystitis $(3,4)$. It has also been elucidated that HMTA is well tolerated, even at doses of up to $5 \mathrm{~g} / \mathrm{kg} /$ day $(4,13)$. Previous studies have shown that DXR can react with formaldehyde to yield an activated form of DXR that can further react with DNA to yield DXR-DNA adducts (4). Because HMTA is known to hydrolyze under cellular conditions and release six molecules of formaldehyde in a pH-dependent manner, we examined HMTA for its potential as a formaldehyde-releasing prodrug for the activation of DXR (4).

Based on our previous study (6) that the intratumor Q cell population includes a much larger hypoxic fraction, where a low pH environment is often created, than the total cell population, more HMTA could be converted into formaldehyde in Q cell fraction, resulting in significantly larger MN frequencies than in the total cell population when HMTA only was administered (Table I). This also supports a more marked enhancement by HMTA in Q cells than the total cell population when DXR was co-administered (Table III).

PLD is characterized by an extended circulation time and reduced volume of distribution $(1,2)$. The extended circulation time and the ability to extravasate through the leaky vasculature of tumors result in the enhanced delivery of PLD to the tumor site $(1,2)$. Thus, the current study also showed an enhancement ratio of $>2.0$ due to encapsulation into pegylated liposomes (Table II), especially in resting Q cells where a clearer enhancing effect due to the extended circulation time could be observed than in the total cell population including $\mathrm{P}$ cells that have strong uptake potential.

Tumor hypoxia results from either limited oxygen diffusion (chronic hypoxia) or limited perfusion (acute hypoxia, transient hypoxia or ischemic hypoxia) $(5,6)$. Chronically hypoxic tumor cells existing at the edge of the oxygen diffusion zone can be killed by just a single administration of a hypoxic cytotoxin. Acutely hypoxic tumor cells occurring sporadically throughout solid tumors can be killed by a hypoxic cytotoxin during long-term continuous administration. Namely, the long-term continuous administration can kill both chronically and acutely hypoxic tumor cells. Actually, it was already shown that total and Q cell populations in SCC VII tumors are rich in acutely and chronically hypoxic fractions, respectively and that the continuous administration of the hypoxic cytotoxin tirapazamine is very useful for sensitizing tumor cells in vivo (7). Therefore, continuously administered HMTA was also more cytotoxic to tumor cells in vivo than a single intraperitoneal administration because the sensitizing effect on the acutely hypoxia-rich total cell population was added to the effect on the chronically hypoxia-rich Q cell population. This is also why the enhancement due to continuous administration compared with a single administration was more marked in the total than $\mathrm{Q}$ cell population (Table III). Furthermore, in both populations, the enhancement in combination with HMTA was greater for free DXR than PLD because the enhancement itself by the encapsulation of DXR into pegylated liposomes was essentially very powerful (Tables II and III).

Basically, the enhancement by the encapsulation of DXR into pegylated liposomes and in combination with HMTA through single or continuous administration was more dramatic in the Q than total cell population. The enhancement in combination with HMTA through continuous administration compared with no combination was more marked in Q cells, although the enhancement compared with a single administration was more remarkable in the total cell population (Table III). Thus, the difference in sensitivity between the total and Q cell populations was reduced with the use of PLD and in combination with HMTA, especially when administered continuously (Table IV). The presence of Q cells is thought to 
be due, in part, to hypoxia and the depletion of nutrition in the tumor core and this is another consequence of poor vascular supply (5). This might promote the formation of micronuclei in Q tumor cells even without the administration of DXR (Table I). Essentially, Q cells showed less sensitivity to DXR, similar to many other conventional chemotherapeutic agents (6). This means that more $Q$ cells can survive after conventional chemotherapy than $\mathrm{P}$ cells. Thus, the control of $\mathrm{Q}$ cells has a great impact on the outcome of anti-cancer chemotherapy. Taking into account that HMTA has a history of clinical use as an antiseptic for urinary tract infections $(4,13)$, it may have the potential to be employed together with conventional chemotherapy. However, studies on the toxicity of HMTA in normal tissues still have to be carried out for safety assurance. In terms of the tumor cell-killing effect as a whole, including intratumor Q cell control, encapsulation of a chemotherapeutic agent into pegylated liposomes and the combined use of continuously administered HMTA may be a promising treatment modality for refractory tumors because of the efficient cytotoxic effects.

\section{Acknowledgements}

This study was supported, in part, by a Grant-in-aid for Scientific Research (C) (20591493) from the Japan Society for the Promotion of Science.

\section{References}

1. Vail DM, Amantea MA, Colbern GT, Martin FJ, Hilger RA and Working PK: Pegylated liposomal doxorubicin: proof of principle using preclinical animal models and pharmacokinetic studies. Semin Oncol 31 (Suppl 13): 16-35, 2004.

2. Gabison A, Shmeeda H and Barenholz Y: Pharmacokinetica of pegylated liposomal doxorubicin: review of animal and human studies. Clin Pharmacokinet 42: 419-436, 2003.
3. Ridpath JR, Nakamura J, Tano K, et al: Cells deficient in the Fanc/Brac pathway are hypersensitive to plasma levels of formaldehyde. Cancer Res 67: 11117-11122, 2007.

4. Swift LP, Cutts SM, Rephaeli A, Nudelman A and Phillips DR: Activation of adriamycin by the $\mathrm{pH}$-dependent formaldehydereleasing prodrug hexamethylenetetramine. Mol Cancer Therapeut 2: 189-198, 2003.

5. Vaupel P: Tumor microenvironmental physiology and its implications for radiation oncology. Semin Radiat Oncol 14: $197-275,2004$

6. Masunaga S and Ono K: Significance of the response of quiescent cell populations within solid tumors in cancer therapy. J Radiat Res 43: 11-25, 2002.

7. Masunaga S, Nagasawa H, Uto Y, et al: The usefulness of continuous administration of hypoxic cytotoxin combined with mild temperature hyperthermia, with reference to effects on quiescent tumour cell populations. Int J Hyperthermia 21: 305-318, 2005.

8. Masunaga S, Ono K, Suzuki M, et al: Radiosensitization effect by combination with paclitaxel in vivo including the effect on intratumor quiescent cells. Int J Radiat Oncol Biol Phys 50: 1063-1072, 2001.

9. Allen TM, Hansen C, Martin FJ, Redemann C and Yau-Young A: Liposomes containing a systemic lipid derivative of polyethylene glycol show prolonged circulation half-lives in vivo. Biochem Biophys Acta 1066: 29-36, 1991.

10. Masunaga S, Tano K, Watanabe M, et al: Evaluation of the potential of hexamethylenetetramine as a combined agent with $\gamma$-ray irradiation and cisplatin treatment in vivo, compared with tirapazamine. Br J Radiol (In press).

11. Hubal EA, Schlosser PM, Conolly RB and Kimbell JS: Comparison of inhaled formaldehyde dosimetry predictions with DNA-protein cross-link measurements in the rat nasal passages. Toxicol Appl Pharmacol 143: 47-55, 1997.

12. Kim JW and Dang CV: Cancer's molecular sweet tooth and the Warburg effect. Cancer Res 66: 8927-8930, 2006.

13. Iskandarova GT: Hygienic rationale for maximum permissible concentration of hexamethylenetetramine salt of 2-chloroethylphosphonic acid. Gig Sanit 10: 14-17, 1993. 